\title{
100 Years of the Tohoku Journal of Experimental Medicine, and the Tohoku Medical Megabank Project in the Japanese New Era, Reiwa
}

\author{
Kazuhiro Takahashi ${ }^{1}$ and Shigeki Shibahara ${ }^{2}$ \\ ${ }^{1}$ Executive Editor, Tohoku Journal of Experimental Medicine, Sendai, Miyagi, Japan \\ ${ }^{2}$ Editor-in-Chief, Tohoku Journal of Experimental Medicine, Sendai, Miyagi, Japan
}

Tohoku J. Exp. Med., 2019 May, 248 (1), 1-2. C 2019 Tohoku University Medical Press

In 1920, the Tohoku Journal of Experimental Medicine (TJEM) was founded by professors of Tohoku Imperial University, Medical School (Shibahara 2009, 2010). The three founding editors were Toshihiro Fujita (Physiology), Toyoziro Kato (Internal Medicine) and Yasutaka Satake (Physiology). The first Issue of the TJEM was published on April 29, 1920, and then, the TJEM has been published continuously, except for the year of 1946 just after the World War II. The TJEM has just entered 100th year of the publication, and will celebrate its $100^{\text {th }}$ anniversary of the founding in 2020 .

The representative historic articles published by the TJEM during 100 years include the study on the endocrine function of pancreas by Kumagai and Osato (1920), the case report on Chédiak-Higashi syndrome by Higashi (1954), the case report on insulin autoimmune syndrome by Hirata and Ishizu (1972), and the establishment of the rat model of type 2 diabetes mellitus, Goto-Kakizaki rats (Goto et al. 1976). Moreover, Hans Selye, the famous founder of the stress theory, published two original articles in the TJEM (Selye 1958; Ishii et al. 1961).

On March 11, 2011, the Great East-Japan Earthquake (magnitude 9.0) attacked the Tohoku region (northeastern Japan), which included Sendai City (Shibahara 2011, 2012; Ishigaki et al. 2013). The earthquake generated a devastating tsunami, leading to unprecedented disasters $(\sim 18,500$ victims) in coastal areas of Iwate, Miyagi and Fukushima prefectures. Moreover, the Great East-Japan Earthquake caused the Fukushima Nuclear Accident, which spread radioactive materials and produced both internal and external exposure.

In April 2011, just after the Great East Japan Earthquake, the TJEM added the field of disaster prevention sciences including earthquake archeology to its subject area (Shibahara 2011). The TJEM is now open to original articles in all branches of medical sciences and relevant areas, including clinical medicine, basic/translational medicine, social medicine, nursing sciences, and disaster prevention sciences. The recent publication of the TJEM included the reports on sleep disturbance among survivors of the Great East Japan Earthquake (Yabe et al. 2018), and psychological problems after the Fukushima Nuclear Accident (Hino et al. 2016; Murakami et al. 2019), and the backup system for patient clinical information against disaster (Ido et al., 2019).

The Tohoku Medical Megabank (TMM) Project is a reconstruction program from the Great East Japan Earthquake and Tsunami, and was funded and started in February 2012 (Minegishi et al. 2019). The aim of the project is to establish two large population cohorts; a TMM community cohort study and a TMM birth-and-three-generation cohort study, and a biobank based on these two cohorts. The TMM biobank is the largest population-based biobank in Japan, covering a wide range of generations, and will be able to generate an important infrastructure for the realization of next-generation medicine.

In May 2019, we welcome the new Japanese Era, Reiwa. Reiwa means 'beautiful harmony' according to the Ministry of the Foreign Affairs of Japan. In this first Issue of the TJEM in the Reiwa Era, we are very pleased to publish an invited review article on the Tohoku Medical Megabank Project by Minegishi et al. (2019), and start to celebrate 100 years of the TJEM. The invited review series on various topics for celebrating 100 years of the TJEM will continue until the end of 2020 .

\section{References}

Goto, Y., Kakizaki, M. \& Masaki, N. (1976) Production of spontaneous diabetic rats by repetition of selective breeding. Tohoku J. Exp. Med., 119, 85-90.

Higashi, O. (1954) Congenital gigantism of peroxidase granules. Tohoku J. Exp. Med., 59, 315-332.

Published online May 23, 2019; doi: 10.1620/tjem.248.1.

Correspondence: Kazuhiro Takahashi, M.D., Ph.D., Department of Endocrinology and Applied Medical Science, Tohoku University Graduate School of Medicine, 2-1 Seiryo-machi, Aoba-ku, Sendai, Miyagi 980-8575, Japan.

e-mail: ktaka-md@med.tohoku.ac.jp 
Hino, Y., Murakami, M., Midorikawa, S., Ohtsuru, A., Suzuki, S., Tsuboi, K. \& Ohira, T. (2016) Explanatory meetings on thyroid examination for the "Fukushima Health Management Survey" after the Great East Japan Earthquake: reduction of anxiety and improvement of comprehension. Tohoku J. Exp. Med., 239, 333-343.

Hirata, Y. \& Ishizu, H. (1972) Elevated insulin-binding capacity of serum proteins in a case with spontaneous hypoglycemia and mild diabetes not treated with insulin. Tohoku J. Exp. Med., 107, 277-286.

Ido, K., Nakamura, N. \& Nakayama, M. (2019) Miyagi Medical and Welfare Information Network: a backup system for patient clinical information after the Great East Japan Earthquake and tsunami. Tohoku J. Exp. Med., 248, 19-25.

Ishigaki, A., Higashi, H., Sakamoto, T. \& Shibahara, S. (2013) The Great East-Japan Earthquake and devastating tsunami: an update and lessons from the past Great Earthquakes in Japan since 1923. Tohoku J. Exp. Med., 229, 287-299.

Ishii, T., Jean, P. \& Selye, H. (1961) Role of chemical elements in eliciting experimental cutaneous calcinosis, following pretreatment with dihydrotachysteroll. Tohoku J. Exp. Med., 74, 235-241.

Kumagai, T. \& Osato, S. (1920) Experimentelles studium der inneren sekretion des pankreas. Tohoku J. Exp. Med., 1, 153-166.

Minegishi, N., Nishijima, I., Nobukuni, T., Kudo, H., Ishida, N., Terakawa, T., Kumada, K., Yamashita, R., Katsuoka, F., Ogishima, S., Suzuki, K., Sasaki, M., Satoh, M., Tohoku
Medical Megabank Project Study Group \&Yamamoto, M. (2019) Biobank establishment and sample management in the Tohoku Medical Megabank Project. Tohoku J. Exp. Med., 248, 45-55.

Murakami, M., Takebayashi, Y. \& Tsubokura, M. (2019) Lower psychological distress levels among returnees compared with evacuees after the Fukushima Nuclear Accident. Tohoku J. Exp. Med., 247, 13-17.

Selye, H. (1958) "Infarctoid cardiopathy" elicited by temporary obstruction of the pylorus in humorally conditioned animals. Tohoku J. Exp. Med., 67, 335-340.

Shibahara, S. (2009) The upcoming 90th anniversary of the Tohoku Journal of Experimental Medicine. Tohoku J. Exp. Med., 218, 161-163.

Shibahara, S. (2010) Sensing the soul of science via Sendai: the prologue to the tenth decade of TJEM. Tohoku J. Exp. Med., 220, $1-2$.

Shibahara, S. (2011) The 2011 Tohoku earthquake and devastating tsunami. Tohoku J. Exp. Med., 223, 305-307.

Shibahara, S. (2012) Revisiting the March 11, 2011 earthquake and tsunami: resilience and restoration. Tohoku J. Exp. Med., 226, 1-2.

Yabe, Y., Hagiwara, Y., Sekiguchi, T., Sugawara, Y., Tsuchiya, M., Koide, M., Itaya, N., Yoshida, S., Sogi, Y., Yano, T., Tsuji, I. \& Itoi, E. (2018) Sleep disturbance is associated with new onset and continuation of lower back pain: a longitudinal study among survivors of the Great East Japan Earthquake. Tohoku J. Exp. Med., 246, 9-14. 